\title{
Integral LOS Control for Path Following of Underactuated Marine Surface Vessels in the Presence of Constant Ocean Currents
}

\author{
Even Børhaug, A. Pavlov and Kristin Y. Pettersen \\ Norwegian University of Science and Technology, NO-7491 Trondheim, Norway.
}

\begin{abstract}
In this paper, we consider the development of a control strategy for path following of underactuated marine surface vessels in the presence of ocean currents. The proposed control strategy is based on a modified Line-of-Sight (LOS) guidance law with integral action and a pair of adaptive feedback controllers. Traditional LOS guidance has several nice properties and is widely used in practice for path following of marine vehicles. However, it has the drawback of being susceptible to environmental disturbances. In this work, we propose a modified LOS guidance law with integral action for counteracting environmental disturbances. Paired with a set of adaptive feedback controllers, we show that this approach guarantees global asymptotic path following of straight-line paths in the presence constant and irrotational ocean currents.
\end{abstract}

\section{INTRODUCTION}

In this paper, we consider the problem of path following of underactuated marine surface vessels in the presence of ocean currents. In particular, we consider the problem of following a straight-line path with a desired speed in the presence of constant, irrotational ocean currents.

Path following control methods based on the Line-of-Sight (LOS) guidance principle are widely used (see e.g. [1-5]) and LOS guidance has some nice properties. In particular, LOS guidance is designed to mimic the actions of a helmsman, which will typically steer the vessel towards a point lying a constant distance, called the look-ahead distance, ahead of the vessel, along the desired path. LOS guidance is simple, intuitive and easy to tune and gives, in the case of no environmental disturbances, nice path convergence properties. However, path following control approaches based on traditional LOS guidance are susceptible to environmental disturbances such as ocean currents, waves and wind. In particular, path deviation and convergence problems will occur if the vessel is affected by environmental disturbances. Moreover, this drawback cannot be fixed by simply adding integral action to the heading controller, i.e. by using a PID controller, since the source of the problem originates from the heading reference generator, that is, the LOS guidance law itself.

The main contribution of this paper is a new guidance law with integral action. The proposed guidance law is based on the LOS guidance principle, but includes integral action to counteract environmental disturbances. The new guidance law overcomes the above mentioned drawbacks of traditional LOS guidance, while preserving the intuition and the simplicity of traditional LOS guidance. For this reason, the guidance law presented in this paper is applicable to a wide range of systems and not just marine surface vessels. In this paper, however, we study the particular problem for marine surface vessels. In particular, we pair the proposed guidance law with a set of adaptive tracking controllers and prove that the proposed control approach gives global asymptotic path following in the presence of constant, irrotational ocean currents. We study the problem both at the kinematic and dynamic level and provide explicit bounds on the parameters of the proposed guidance law.

This paper is organized as follows. In Section II, we present the system model and state the control problem to be solved. In Section III we review traditional LOS guidance and in Section IV we present the controllers that solve the control problem stated in Section II. In Section V we formulate the main technical result of the paper and present the proof of this result in Section VI. Conclusions are given in Section VII.

\section{System Model And Control ObJective}

In this section, we present the mathematical model of the class of vessels considered in this paper. Moreover, we introduce notation to be used throughout the paper and formulate the control problem to be solved.

\section{A. Vessel Model}

We consider the class of marine surface vessel that can be described by the 3 -DOF model ([6]):

$$
\begin{aligned}
\dot{x} & =u \cos \psi-v \sin \psi \\
\dot{y} & =u \sin \psi+v \cos \psi \\
\dot{\psi}= & r, \\
\boldsymbol{M}_{R B} \dot{\boldsymbol{\nu}}+\boldsymbol{C}_{R B}(\boldsymbol{\nu}) \boldsymbol{\nu}= & -\boldsymbol{M}_{A} \dot{\boldsymbol{\nu}}_{r}-\boldsymbol{C}_{A}\left(\boldsymbol{\nu}_{r}\right) \boldsymbol{\nu}_{r} \\
& -\boldsymbol{D} \boldsymbol{\nu}_{r}+\boldsymbol{B} \boldsymbol{f} .
\end{aligned}
$$

Here, $(x, y, \psi)$ is the position and orientation of the vessel with respect to an inertial reference frame $i$ and $\nu \triangleq[u, v, r]^{T}$ is a vector of body-fixed velocities, where $u$ is the surge velocity, $v$ is the sway velocity and $r$ is the yaw angular velocity, respectively. Moreover, $\boldsymbol{\nu}_{r} \triangleq \boldsymbol{\nu}-\boldsymbol{\nu}_{c}$ is the relative velocity of the vehicle, where $\boldsymbol{\nu}_{c}=\boldsymbol{R}^{T}(\psi) \boldsymbol{v}_{c} \triangleq\left[u_{c}, v_{c}, 0\right]^{T}$ is the ocean current velocity expressed in body frame coordinates and $\boldsymbol{v}_{c} \triangleq\left[V_{x}, V_{y}, 0\right]^{T}$ is the ocean current velocity expressed in inertial coordinates. The inertial ocean current $\boldsymbol{v}_{c}$ is assumed to be constant and irrotational, i.e. $\dot{\boldsymbol{v}}_{c}=\mathbf{0}$. This gives that

$$
\dot{\boldsymbol{\nu}}_{c}=\frac{d}{d t}\left(\boldsymbol{R}^{T}(\psi) \boldsymbol{v}_{c}\right)=\left[r v_{c},-r u_{c}, 0\right]^{T} .
$$

The vector $f \in \mathbb{R}^{2}$ is the control input vector. Notice that the model (1d) is underactuated, since the dimension 
of the control input vector $f$ is one less than the system dimension. Furthermore, the matrix $\boldsymbol{M}_{R B}=\boldsymbol{M}_{R B}^{T}>0$ is the rigid-body mass and inertia matrix, $C_{R B}$ is the rigidbody Coriolis and centripetal matrix, $\boldsymbol{M}_{A}=\boldsymbol{M}_{A}^{T}>0$ is the hydrodynamic added mass matrix, $\boldsymbol{C}_{A}$ is the added mass Coriolis and centripetal matrix, $\boldsymbol{D}$ is the hydrodynamic damping matrix and $\boldsymbol{B} \in \mathbb{R}^{3 \times 2}$ is the actuator configuration matrix.

The system matrices $\boldsymbol{M}_{R B}, \boldsymbol{M}_{A}, \boldsymbol{D}$ and $\boldsymbol{B}$ are assumed to have the following structure:

$$
\begin{aligned}
\boldsymbol{M}_{x} & \triangleq\left[\begin{array}{ccc}
m_{11}^{x} & 0 & 0 \\
0 & m_{22}^{x} & m_{23}^{x} \\
0 & m_{23}^{x} & m_{33}^{x}
\end{array}\right], \quad x \in\{R B, A\}, \\
\boldsymbol{D} & \triangleq\left[\begin{array}{ccc}
d_{11} & 0 & 0 \\
0 & d_{22} & d_{23} \\
0 & d_{32} & d_{33}
\end{array}\right], \boldsymbol{B} \triangleq\left[\begin{array}{cc}
b_{11} & 0 \\
0 & b_{22} \\
0 & b_{32}
\end{array}\right],
\end{aligned}
$$

where $\boldsymbol{M}_{x}=\boldsymbol{M}_{x}^{T}>0$. To obtain the particular structure of the system matrices given in (3), we have assumed that the vessel is port-starboard symmetric (most vessels are) and that the body-fixed coordinate system, which we can freely choose the position and orientation of, is located along the centerline of the vessel (see [6]). With the above given structure for $M_{R B}$ and $\boldsymbol{M}_{A}$, the Coriolis and centripetal matrices $\boldsymbol{C}_{R B}$ and $C_{A}$ can be parameterized as (see [6]):

$$
\boldsymbol{C}_{x}(\boldsymbol{z}) \triangleq\left[\begin{array}{ccc}
0 & 0 & c_{13}\left(z_{2}, z_{3}\right) \\
0 & 0 & c_{23}\left(z_{1}\right) \\
-c_{13}\left(z_{2}, z_{3}\right) & -c_{23}\left(z_{1}\right) & 0
\end{array}\right],
$$

where $x \in\{R B, A\}$ and

$$
c_{13}\left(z_{2}, z_{3}\right) \triangleq-m_{22}^{x} z_{2}-m_{23}^{x} z_{3}, \quad c_{23}\left(z_{1}\right) \triangleq m_{11}^{x} z_{1} .
$$

Without loss of generality, we assume in the following that the body-fixed coordinate system is located in a point $\left(x_{g}^{*}, 0\right)$ along the centerline of the vessel, where $x_{g}^{*}$ is such that $\boldsymbol{M}^{-1} \boldsymbol{B} \boldsymbol{f}=\left[\tau_{u}, 0, \tau_{r}\right]^{T}$. Here, $\boldsymbol{M} \triangleq \boldsymbol{M}_{R B}+\boldsymbol{M}_{A}$. Note that if the body-fixed coordinate system is not originally located in $x_{g}^{*}$, but in some other point $x_{g}$ along the center line of the vessel, then the coordinate system can easily be translated to the required location. The relevant coordinate transformations are given in [5].

By inserting (2) in (1d) and multiplying from the left by $\boldsymbol{M}^{-1}$, we obtain the following set of system equations:

$$
\begin{aligned}
\dot{u} & =-\frac{d_{11}}{m_{11}} u+\frac{\left(m_{22} v+m_{23} r\right) r}{m_{11}}+\boldsymbol{\phi}_{u}^{T}(\psi, r) \boldsymbol{\theta}_{u}+\tau_{u} \\
\dot{v} & =X\left(u_{r}, u_{c}\right) r+Y\left(u_{r}\right) v_{r} \\
\dot{r} & =F_{r}(u, v, r)+\boldsymbol{\phi}_{r}^{T}(u, v, r, \psi) \boldsymbol{\theta}_{r}+\tau_{r} .
\end{aligned}
$$

Here, $m_{i j} \triangleq m_{i j}^{R B}+m_{i j}^{A}, \boldsymbol{\theta}_{u} \triangleq\left[V_{x}, V_{y}\right]^{T}$ and $\boldsymbol{\theta}_{r} \triangleq\left[V_{x}, V_{y}, V_{x}^{2}, V_{y}^{2}, V_{x} V_{y}\right]^{T}$. The expressions for $\boldsymbol{\phi}_{u}^{T}(\psi, r)$, $X\left(u_{r}, u_{c}\right), Y\left(u_{r}\right), F_{r}(u, v, r)$ and $\phi_{r}^{T}(u, v, r, \psi)$ are given in Appendix A.

Remark: In many earlier works on control in the presence of ocean currents, the current is assumed to be constant in the body frame, i.e. $\dot{\boldsymbol{\nu}}_{c}=\mathbf{0}$. Note however that this assumption is easily violated during turning, cf. Eq. (2). In this paper, we instead make the assumption that the ocean current is constant in the inertial frame, i.e. that $\dot{\boldsymbol{v}}_{c}=\mathbf{0}$, which is a more natural assumption.

\section{B. Control Objective}

The goal of this paper is to design a control system for the vessel, which dynamics are described by (6), such that starting from any location, the vessel converges to and follows a given straight-line path $\mathcal{P}$ with a desired constant speed $U_{d}>0$. This statement should hold also in the presence of an unknown, constant and irrotational inertial ocean currents $\boldsymbol{v}_{c}=\left[V_{x}, V_{y}, 0\right]^{T}$. To this end, we place the inertial coordinate system $i$ with the $x$-axis along the desired path. With this choice, the desired path is given by $\mathcal{P} \triangleq\left\{(x, y) \in \mathbb{R}^{2}: y=0\right\}$ and the $y$-position of the vessel can be seen as the cross-track error, i.e. the minimum distance from the vessel to the desired path. With this notation, the control objective can be formalized as

$$
\begin{aligned}
& \lim _{t \rightarrow \infty} y(t)=0, \\
& \lim _{t \rightarrow \infty} \psi(t)=\psi_{s s}, \quad \psi_{s s} \in(-\pi / 2, \pi / 2), \\
& \lim _{t \rightarrow \infty} U(t)=U_{d} .
\end{aligned}
$$

Here, $U \triangleq \sqrt{u(t)^{2}+v(t)^{2}}$ is the total speed of the vessel and $\psi_{s s}$ is a constant.

Remark: Note that we do not require that $\psi(t) \rightarrow 0$ as $t \rightarrow \infty$, but rather that $\psi$ converges to a constant value $\psi_{s s}$, where $\psi_{s s} \in(-\pi / 2, \pi / 2)$. In the presence of currents, the vessel must be allowed to side-slip in order for a component of the velocity to counteract the current. As a result of this, the vessel will have a non-zero heading in steady-state. Our control objective is to control the heading of the vessel such that, in steady-state, the vessel counteracts the currents and precisely follows the desired path (see Fig. 1).

\section{TRaditional LOS Guidance}

In this section, we briefly review traditional Line-Of-Sight (LOS) guidance for path following and discuss some of its nice features and some of its drawbacks.

In traditional LOS guidance, the desired heading of the vessel is given by the guidance law

$$
\psi_{L O S} \triangleq-\tan ^{-1}\left(\frac{y}{\Delta}\right), \quad \Delta>0 .
$$

The angle $\psi_{L O S}$ is called the LOS angle and, geometrically, it corresponds to the angle between the path $y=0$ and the line-of-sight from the vessel to a point lying a distance $\Delta>0$ ahead of the vessel, along the path $y=0$. By making the yaw angle $\psi$ of the vessel track the LOS angle $\psi_{L O S}$ and by maintaining a non-zero forward speed, one can show, under certain assumptions, that the vessel (6) converges to and follows the path $y=0$ (see e.g. [5]). However, this statement is only true if there are no ocean currents or other environmental disturbances. The reason for this is that $\psi_{L O S}=0$ whenever $y=0$. This means that the LOS guidance law (10) wants the vessel to move along the path $y=0$ with zero heading, i.e. $\psi=0$. However, if the ocean 
current has a component acting in the direction normal to the path, an underactuated vessel such as (6) cannot stay identically on the path $y=0$ with zero heading. This is a major drawback that results in deviation problems when using LOS control in the presence of disturbances. Instead the vessel must be allowed to side-slip such that a component of the forward velocity of the vessel can counteract the effect of the ocean current. In particular, this implies that the vessel must have a non-zero heading in order to move along the path, see Fig. 1. This is the motivation for this paper: to develop an improved LOS design that handles ocean currents by automatically creating the reference heading angle that produces the necessary side-slip.

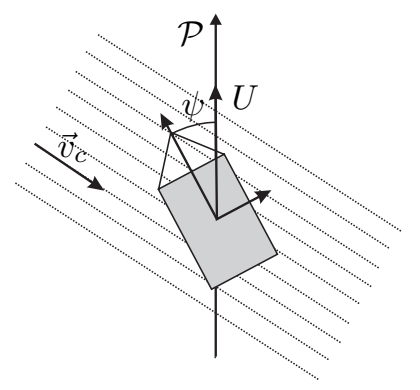

Fig. 1. Path following in the presence of currents.

\section{CONTROL SYSTEM}

In this section, we present the control system that solves the control problem stated in Section II-B. The proposed control strategy consists of three components: a modified LOS guidance law with integral action, an adaptive yaw tracking controller for tracking the desired heading provided by the guidance law and an adaptive surge tracking controller for tracking a desired surge speed profile. In this section, we present the control laws and analyze the closed loop tracking error dynamics. In Section V, we provide the conditions under which the proposed control strategy meets the control goals (7)-(9). Before we present the controllers, we state the assumptions on which the controller design will be based.

\section{A. Assumptions}

The controller design presented and analyzed in the next sections will be based on the following assumptions:

A.1: The ocean current is irrotational and constant in the inertial frame, i.e. $\boldsymbol{v}_{c}=\left[V_{x}, V_{y}, 0\right]^{T}$, where $\dot{\boldsymbol{v}}_{c}=\mathbf{0}$.

A.2: The ocean current intensity has a known upper bound $V_{\max }>0$, i.e.

$$
\left\|\boldsymbol{v}_{c}\right\|_{2}=\sqrt{V_{x}^{2}+V_{y}^{2}} \leq V_{\max }<\infty .
$$

A.3: The desired constant speed $U_{d}$ is strictly greater than the ocean current intensity, i.e. $U_{d}>V_{\max }$.

A.4: The function $Y\left(u_{r}\right)$ satisfies

$$
Y\left(u_{r}\right) \leq-Y_{\min }<0, \quad \forall u_{r} \in\left[-V_{\max }, U_{d}+V_{\max }\right] .
$$

Assumption A.1 is a simplification of the real world situation. Assumption A.2 will be used when deriving stability criteria and Assumption A.3 is necessary to avoid that the vessel looses control and drifts away with the current. Assumption A.4 is a natural assumption for marine surface vessels, since $Y>0$ would imply that the vessel is open-loop unstable in sway, which is not the case in practice.

Remark: Note that a marine surface vessel can be openloop unstable in sway-yaw, e.g. course unstable vessels. However, a vessel cannot be open-loop unstable in the sense that $Y\left(u_{r}\right)>0$, since this would imply that the system is not passive. A small perturbation in sway would then result in an accelerating sway velocity, which is not a realistic response for a marine vessel.

\section{B. Integrative LOS Guidance}

As discussed in Section III, traditional LOS guidance is simple and intuitive and has several nice properties. However, traditional LOS guidance is not designed to handle ocean currents or any other environmental disturbances such as wind or waves. To this end, we propose a modified LOS guidance law with integral action:

$$
\begin{aligned}
\psi_{L O S}^{m} & \triangleq-\tan ^{-1}\left(\frac{y+\sigma y_{\text {int }}}{\Delta}\right), \quad \Delta>0 . \\
\dot{y}_{\text {int }} & =\frac{\Delta y}{\left(y+\sigma y_{\text {int }}\right)^{2}+\Delta^{2}} .
\end{aligned}
$$

Here, $\sigma>0$ is a design parameter, an integral gain, and $\Delta$ has the same interpretation as in the case of traditional LOS guidance, see Section III. The idea behind (11) is that the integral of the cross-track error $y$ will allow $\psi_{L O S}^{m}$ to be non-zero when $y=0$, i.e. when the vessel is on the desired path. In particular, in the presence of disturbances driving the system away from its path, the integral of the cross-track error $y$ will build up to create a non-zero $\psi_{L O S}^{m}$. When the vessel is on the desired path, the integral term will generate the necessary side-slip angle to follow the path. These statements will be made clear in Section V.

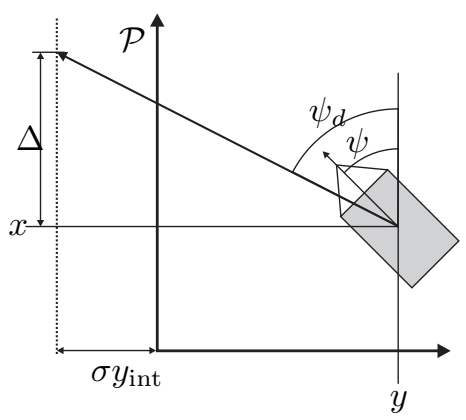

Fig. 2. Illustration of modified LOS guidance.

Remark: Note that Eq. (11b) has the property that $\dot{y}_{\text {int }} \rightarrow$ 0 as $y \rightarrow \infty$. This means that the rate of integration will decrease with large cross-track errors. In particular, the integral term will be less dominant when the cross-track error is large, i.e. when the vessel is far from the desired path. This property will reduce the risk of integrator wind-up and reduce performance limitations related to integrator wind-up. 


\section{Yaw Control}

To guide the vessel towards the desired path, we control the yaw angle $\psi$ of the vessel to the desired yaw angle $\psi_{d} \triangleq$ $\psi_{L O S}^{m}$, where $\psi_{L O S}^{m}$ is given by the modified LOS guidance law (11). This is done using the adaptive yaw controller

$$
\begin{aligned}
\tau_{r}= & -F_{r}(u, v, r)-\phi_{r}^{T}(u, v, r, \psi) \hat{\boldsymbol{\theta}}_{r}+\ddot{\psi}_{d} \\
& \quad-\left(k_{\psi}+\lambda k_{r}\right)\left(\psi-\psi_{d}\right)-\left(k_{r}+\lambda\right)\left(r-\dot{\psi}_{d}\right), \\
\dot{\hat{\boldsymbol{\theta}}}_{r}= & \gamma_{r} \boldsymbol{\phi}_{r}(u, v, r, \psi)\left[\left(r-\dot{\psi}_{d}\right)+\lambda\left(\psi-\psi_{d}\right)\right] .
\end{aligned}
$$

Here, $\lambda, k_{\psi}, k_{r}>0$ are constant controller gains and $\gamma_{r}>$ 0 is a constant adaptation gain. As follows from Eq. (6c), the controller (12) is an adaptive feedback linearizing PDcontroller. Note that the controller (12) does not depend on the relative velocities, which are not available for feedback.

We define the errors $\tilde{\psi} \triangleq \psi-\psi_{d}, s \triangleq \dot{\tilde{\psi}}+\lambda \tilde{\psi}$ and $\tilde{\boldsymbol{\theta}}_{r} \triangleq$ $\hat{\boldsymbol{\theta}}_{r}-\boldsymbol{\theta}_{r}$. Taking the time-derivative of $\tilde{\psi}, s$ and $\tilde{\boldsymbol{\theta}}_{r}$, and using (1c), (6c), (12a) and (12b), then gives

$$
\begin{aligned}
{\left[\begin{array}{c}
\dot{\tilde{\psi}} \\
\dot{s}
\end{array}\right] } & =\left[\begin{array}{cc}
-\lambda & 1 \\
-k_{\psi} & -k_{r}
\end{array}\right]\left[\begin{array}{c}
\tilde{\psi} \\
s
\end{array}\right]-\left[\begin{array}{c}
\mathbf{0}^{T} \\
\boldsymbol{\phi}_{r}^{T}
\end{array}\right] \tilde{\boldsymbol{\theta}}_{r} . \\
\dot{\tilde{\boldsymbol{\theta}}}_{r} & =\gamma_{r} \boldsymbol{\phi}_{r}(u, v, r, \psi) s .
\end{aligned}
$$

To analyze the stability properties of the interconnected system (13), we consider the time-derivative of the positive definite and radially unbounded Lyapunov function candidate (LFC) $V_{\psi} \triangleq 1 / 2 k_{\psi} \tilde{\psi}^{2}+1 / 2 s^{2}+1 /\left(2 \gamma_{r}\right) \tilde{\boldsymbol{\theta}}_{r}^{T} \tilde{\boldsymbol{\theta}}_{r}$ along the solutions of system (13). This gives

$$
\dot{V}_{\psi}=-\lambda k_{\psi} \tilde{\psi}^{2}-k_{r} s^{2} \leq 0
$$

From Eq. (14) is follows that $\dot{V}_{\psi}$ is negative semi-definite on $\mathbb{D}_{\psi}=\mathbb{R}^{2} \times \mathbb{R}^{5}$. It hence follows, from standard Lyapunov arguments, that the origin $\left(\tilde{\psi}, s, \tilde{\boldsymbol{\theta}}_{r}\right)=(0,0, \mathbf{0})$ is a uniformly globally stable (UGS) equilibrium of the interconnected system (13). Note that system (13) is non-autonomous. Hence, LaSalle's invariance principle is not applicable in this case. However, it is straightforward to apply, for example Barbalat's Lemma, to conclude that $(\tilde{\psi}, s) \rightarrow(0,0)$ asymptotically as $t \rightarrow \infty$ and that $\tilde{\psi}(t), s(t) \in \mathcal{L}_{2}$. Note that we cannot hope to show that the equilibrium $\left(\tilde{\psi}, s, \tilde{\boldsymbol{\theta}}_{r}\right)=(0,0, \mathbf{0})$ is UGAS, since the regressor $\phi_{r}(u, v, r, \psi)$ is not persistently exciting (PE). A persistently exciting regressor is a necessary condition for UGAS of adaptive systems such as (13) (see e.g. [7]). Finally, note that asymptotic convergence of $\tilde{\psi}$ and $s$ to zero also implies asymptotic convergence of $r$ to $\dot{\psi}_{d}$, since $\left(r-\dot{\psi}_{d}\right)=s-\lambda \tilde{\psi}$ by definition.

\section{Surge Control}

The control goal (9) is to control the total speed $U$ of the vessel to the desired constant value $U_{d}$. To this end, we choose the desired surge speed as

$$
u_{d} \triangleq U_{d} \cos \psi_{d}
$$

where $\psi_{d} \in(-\pi / 2, \pi / 2)$ is the desired heading given by Eq. (11a). The motivation behind (15) will become clear in Section V.
To make $u \rightarrow u_{d}$, we propose the following adaptive surge controller:

$$
\begin{aligned}
& \tau_{u}= {\left[-\frac{1}{m_{11}}\left(m_{22} v+m_{23} r\right) r+\frac{d_{11}}{m_{11}} u_{d}\right.} \\
&\left.-\boldsymbol{\phi}_{u}^{T}(\psi, r) \hat{\boldsymbol{\theta}}_{u}+\dot{u}_{d}-k_{u}\left(u-u_{d}\right)\right], \\
& \dot{\hat{\boldsymbol{\theta}}}_{u}=\gamma_{u} \boldsymbol{\phi}_{u}(\psi, r)\left(u-u_{d}\right) .
\end{aligned}
$$

Here, $k_{u}>0$ is a constant controller gain and $\gamma_{u}>0$ is a constant adaptation gain. As follows from Eq. (6a), the controller (16) is an adaptive feedback linearizing Pcontroller. Similar to the controller (12), the controller (16) does not depend on the relative velocities, which are not available for feedback.

We define the errors $\tilde{u} \triangleq u-u_{d}$ and $\tilde{\boldsymbol{\theta}}_{u} \triangleq \hat{\boldsymbol{\theta}}_{u}-\boldsymbol{\theta}_{u}$. Using (6a), (16a) and (16b), we then derive the closed loop dynamics of the errors $\tilde{u}$ and $\tilde{\boldsymbol{\theta}}_{u}$ :

$$
\begin{aligned}
\dot{\tilde{u}} & =-\left(\frac{d_{11}}{m_{11}}+k_{u}\right) \tilde{u}-\boldsymbol{\phi}_{u}^{T}(\psi, r) \tilde{\boldsymbol{\theta}}_{u}, \\
\dot{\tilde{\boldsymbol{\theta}}}_{u} & =\gamma_{u} \boldsymbol{\phi}_{u}(\psi, r) \tilde{u} .
\end{aligned}
$$

To analyze the stability properties of the interconnected system (17), we consider the time-derivative of the positive definite and radially unbounded LFC $V_{u} \triangleq 1 / 2 \tilde{u}^{2}+$ $1 /\left(2 \gamma_{u}\right) \tilde{\boldsymbol{\theta}}_{u}^{T} \tilde{\boldsymbol{\theta}}_{u}$ along the solutions of system (17). This gives

$$
\dot{V}_{u}=-\left(\frac{d_{11}}{m_{11}}+k_{u}\right) \tilde{u}^{2} \leq 0 .
$$

From Eq. (18) it follows that $\dot{V}_{u}$ is negative semi-definite on $\mathbb{D}_{u}=\mathbb{R} \times \mathbb{R}^{2}$ and it hence follows, from standard Lyapunov arguments, that the origin $\left(\tilde{u}, \tilde{\boldsymbol{\theta}}_{u}\right)=(0, \mathbf{0})$ is a UGS equilibrium point of the interconnected system (17). Moreover, it is again straightforward using, for example, Barbalat's Lemma, to conclude that $\tilde{u}(t) \rightarrow 0$ asymptotically as $t \rightarrow \infty$ and that $\tilde{u}(t) \in \mathcal{L}_{2}$. Again, the equilibrium is not UGAS, since the regressor $\phi_{u}(\psi, r)$ is not PE.

\section{MAIn RESUlT}

In this section, we present the main technical result of the paper. In particular, we state the conditions under which the control goals (7)-(9) are achieved using the control strategy presented in the previous section.

Before we state the main result, we introduce the following notation:

$$
\bar{X}_{\max } \triangleq \max _{\Omega}\left(\left|X\left(u_{r}, u_{c}\right)-u_{c}\right|\right)
$$

where $X\left(u_{r}, u_{c}\right)$ is given by (52b) in Appendix A and

$$
\Omega=\left\{-V_{\max }<u_{r}<\left(U_{d}+V_{\max }\right),\left|u_{c}\right|<V_{\max }\right\} .
$$

With this notation, we now formulate the main technical result of the paper.

Theorem 1. Consider an underactuated vessel described by the dynamic system (6). If Assumptions A.1-A.4 hold, and if 
the look-ahead distance $\Delta$ of the guidance law (11) satisfies the condition

$$
\Delta>\frac{\bar{X}_{\max }}{Y_{\min }}\left(1+\frac{U_{d}+V_{\max }+\sigma}{U_{d}-V_{\max }-\sigma}+\frac{\sigma^{2}}{2}\right),
$$

where the integral gain $\sigma$ satisfies the condition

$$
\frac{\Delta \frac{Y^{\min }}{X_{\max }} \frac{1}{\left(U_{d}+V_{\max }\right)^{2}}}{1+\Delta \frac{Y^{\min }}{\bar{X}_{\max }} \frac{1}{\left(U_{d}+V_{\max }\right)^{2}}}\left(U_{d}-V_{\max }\right)<\sigma<\left(U_{d}-V_{\max }\right),
$$

then the controllers (12) and (16), where $\psi_{d}$ is given by (11a) and $u_{d}$ is given by (15), guarantees that the control goals (7)(9) are achieved. In particular, control goal (8) is achieved with

$$
\psi_{s s}=-\tan ^{-1}\left(\frac{V_{y}}{U_{d}+V_{x}}\right) \triangleq \psi_{s s}^{*}
$$

Proof. The proof of this theorem is given in Section VI.

Remark: Note that it is always possible to satisfy condition (22), since the left-hand side of the inequality is always strictly less than the right-hand side.

Remark: It is interesting to note that in the case of no currents and no integral action in the guidance law, i.e. setting $\sigma=0$ in (11a), then the condition on the look-ahead distance $\Delta$, given in Eq. (21), reduces to the condition found in [8], where the traditional LOS guidance law (10) was used to achieve path following of a straight-line path for the case of no currents.

Remark: The proposed controllers (12) and (16) rely on feedback linearization. If the model parameters have a high degree of uncertainty, other control approaches may be better suited. Handling uncertain parameters in the controllers remain a topic for future work.

\section{PROOF OF THEOREM 1}

We start the proof of Theorem 1 by considering the dynamics of the cross-track error $y$ and the relative sway velocity $v_{r}$. From the analysis presented in Sections IV-C and IV-D, we know that $u \rightarrow u_{d}, \psi \rightarrow \psi_{d}, r \rightarrow \dot{\psi}_{d}$ asymptotically as $t \rightarrow \infty$ and that the error variables $\tilde{\psi}(t), s(t), \tilde{u}(t) \in \mathcal{L}_{2}$. Using Equations (2), (6b) and the expressions $u=u_{d}+\tilde{u}$, $v=v_{r}+v_{c}, \psi=\psi_{d}+\tilde{\psi}$ and $r=\dot{\psi}_{d}+(s-\lambda \tilde{\psi})$, the crosstrack error dynamics (1b) and the dynamics of the relative sway velocity $v_{r}$ can then be written as

$$
\begin{gathered}
\dot{y}=-\left(u_{d}+\tilde{u}\right) \sin \left(\psi_{d}+\tilde{\psi}\right) \\
\quad+\left(v_{r}+v_{c}\right) \cos \left(\psi_{d}+\tilde{\psi}\right) \\
\dot{v}_{r}=\dot{v}-\dot{v}_{c}=X\left(u_{r}, u_{c}\right) r+Y\left(u_{r}\right) v_{r}-\left(-u_{c} r\right) \\
=\bar{X}\left(u_{r}, u_{c}\right)\left(\dot{\psi}_{d}+(s-\lambda \tilde{\psi})\right)+Y\left(u_{r}\right) v_{r} .
\end{gathered}
$$

Here, $\bar{X}\left(u_{r}, u_{c}\right) \triangleq X\left(u_{r}, u_{c}\right)-u_{c}$. Substituting (11a) for $\psi_{d}$ in the above equations, extending the state space with (11b) and factorizing the result with respect to the errors $\tilde{u}, \tilde{\psi}$ and $s$, then gives

$$
\begin{aligned}
& \dot{y}_{\text {int }}= \frac{\Delta y}{\left(y+\sigma y_{\text {int }}\right)^{2}+\Delta^{2}} \\
& \dot{y}=-u_{d} \frac{y+\sigma y_{\text {int }}}{\sqrt{\left(y+\sigma y_{\text {int }}\right)^{2}+\Delta^{2}}}+v_{r} \frac{\Delta}{\sqrt{\left(y+\sigma y_{\text {int }}\right)^{2}+\Delta^{2}}} \\
&+v_{c} \frac{\Delta}{\sqrt{\left(y+\sigma y_{\text {int }}\right)^{2}+\Delta^{2}}} \\
&+\boldsymbol{h}_{y}^{T}\left(u_{d}, v_{r}, v_{c}, \psi_{d}, \boldsymbol{\xi}\right) \boldsymbol{\xi} \\
& \dot{v}_{r}=\bar{X}\left(u_{r}, u_{c}\right) \dot{\psi}_{d}+Y\left(u_{r}\right) v_{r}+\boldsymbol{h}_{v_{r}}^{T}\left(u_{r}, u_{c}, \boldsymbol{\xi}\right) \boldsymbol{\xi} .
\end{aligned}
$$

Here, $\boldsymbol{\xi} \triangleq[\tilde{u}, \tilde{\psi}, s]^{T}$ is a vector of converging error signals and $\boldsymbol{h}_{y}^{T}\left(u_{d}, v_{r}, v_{c}, \psi_{d}, \boldsymbol{\xi}\right) \boldsymbol{\xi}$ and $\boldsymbol{h}_{v_{r}}^{T}\left(u_{r}, u_{c}, \boldsymbol{\xi}\right) \boldsymbol{\xi}$ contain all terms vanishing at $\boldsymbol{\xi}=\mathbf{0}$. The expressions of $\boldsymbol{h}_{y}$ and $\boldsymbol{h}_{v_{r}}$ are given in Appendix A.

Notice that the current component $v_{c}$, expressed in bodyframe coordinates, can be expressed using the constant inertial ocean current components $V_{x}$ and $V_{y}$ according to

$$
v_{c}=-V_{x} \sin \psi+V_{y} \cos \psi
$$

Substituting this expression for $v_{c}$ in (27) and using Equations (11a), (15) and the expression $\psi=\psi_{d}+\tilde{\psi}$, then gives

$$
\begin{gathered}
\dot{y}_{\text {int }}=\frac{\Delta y}{\left(y+\sigma y_{\text {int }}\right)^{2}+\Delta^{2}} \\
\dot{y}=-\Delta\left(U_{d}+V_{x}\right) \frac{y+\sigma y_{\text {int }}}{\left(y+\sigma y_{\text {int }}\right)^{2}+\Delta^{2}} \\
+V_{y} \frac{\Delta^{2}}{\left(y+\sigma y_{\text {int }}\right)^{2}+\Delta^{2}} \\
+v_{r} \frac{\Delta}{\sqrt{\left(y+\sigma y_{\text {int }}\right)^{2}+\Delta^{2}}} \\
\quad+\hat{\boldsymbol{h}}_{y}^{T}\left(U_{d}, v_{r}, v_{c}, \psi_{d}, \boldsymbol{\xi}\right) \boldsymbol{\xi} \\
\dot{v}_{r}=\bar{X}\left(u_{r}, u_{c}\right) \dot{\psi}_{d}+Y\left(u_{r}\right) v_{r}+\boldsymbol{h}_{v_{r}}^{T}\left(u_{r}, u_{c}, \boldsymbol{\xi}\right) \boldsymbol{\xi} .
\end{gathered}
$$

The expression for $\hat{\boldsymbol{h}}_{y} \triangleq\left[\hat{h}_{y 1}, \hat{h}_{y 2}, \hat{h}_{y 3}\right]^{T}$ are given in Appendix A.

It is straightforward to verify that the equilibrium point of system (30), on the manifold $\boldsymbol{\xi}=\mathbf{0}$, is given by

$$
y_{\text {int }}=\frac{\Delta}{\sigma} \frac{V_{y}}{U_{d}+V_{x}} \triangleq y_{\text {int }}^{\mathrm{eq}}, \quad y=0, \quad v_{r}=0 .
$$

Hence, $V_{y}$ can be written in terms of the equilibrium point $y_{\text {int }}^{\text {eq }}$ according to

$$
V_{y}=\frac{\sigma}{\Delta}\left(U_{d}+V_{x}\right) y_{\text {int }}^{\mathrm{eq}} .
$$

To proceed, we define a new set of coordinates

$$
\begin{aligned}
& z_{1} \triangleq y_{\text {int }}-y_{\text {int }}^{\text {eq }} \\
& z_{2} \triangleq y+\sigma z_{1} .
\end{aligned}
$$

Taking the time-derivative of (33a) and (33b) and using (30) 
and (32), we obtain the transformed dynamics

$$
\begin{gathered}
\dot{z}_{1}=\Delta \frac{z_{2}-\sigma z_{1}}{\left(z_{2}+\sigma y_{\text {int }}^{\text {eq }}\right)^{2}+\Delta^{2}} \\
\dot{z}_{2}=-\Delta\left(U_{d}+V_{x}\right) \frac{z_{2}}{\left(z_{2}+\sigma y_{\text {int }}^{\text {eq }}\right)^{2}+\Delta^{2}} \\
\quad+v_{r} \frac{\Delta}{\sqrt{\left(z_{2}+\sigma y_{\text {int }}^{\text {eq }}\right)^{2}+\Delta^{2}}} \\
+\sigma \Delta \frac{z_{2}-\sigma z_{1}}{\left(z_{2}+\sigma y_{\text {int }}^{\text {eq }}\right)^{2}+\Delta^{2}} \\
\quad+\hat{\boldsymbol{h}}_{y}^{T}\left(U_{d}, v_{r}, v_{c}, \psi_{d}, \boldsymbol{\xi}\right) \boldsymbol{\xi} \\
\dot{v}_{r}=\bar{X}\left(u_{r}, u_{c}\right) \dot{\psi}_{d}+Y\left(u_{r}\right) v_{r}+\boldsymbol{h}_{v_{r}}^{T}\left(u_{r}, u_{c}, \boldsymbol{\xi}\right) \boldsymbol{\xi},
\end{gathered}
$$

Now, substituting the expression

$$
\begin{aligned}
\dot{\psi}_{d} & =\frac{d}{d t}\left(-\tan ^{-1}\left(\frac{z_{2}+\sigma y_{\text {int }}^{\mathrm{eq}}}{\Delta}\right)\right) \\
& =-\frac{\Delta}{\left(z_{2}+\sigma y_{\text {int }}^{\mathrm{eq}}\right)^{2}+\Delta^{2}} \dot{z}_{2},
\end{aligned}
$$

in (34c), where $\dot{z}_{2}$ is given by (34b), we finally obtain

$$
\left[\begin{array}{c}
\dot{z}_{1} \\
\dot{z}_{2} \\
\dot{v}_{r}
\end{array}\right]=\boldsymbol{A}\left(z_{2}, u_{r}, u_{c}\right)\left[\begin{array}{c}
z_{1} \\
z_{2} \\
v_{r}
\end{array}\right]+\boldsymbol{H}\left(t, z_{2}, v_{r}, \boldsymbol{\xi}\right) \boldsymbol{\xi},
$$

where $\boldsymbol{A}\left(z_{2}, u_{r}, u_{c}\right)$ is given by Eq. (38) and

$$
\boldsymbol{H}\left(t, z_{2}, v_{r}, \boldsymbol{\xi}\right) \triangleq\left[\begin{array}{c}
0 \\
\hat{\boldsymbol{h}}_{y}^{T} \\
\boldsymbol{h}_{v_{r}}^{T}-\frac{\Delta X\left(u_{r}, u_{c}\right)}{\left(z_{2}+\sigma y_{\mathrm{int}}^{\mathrm{eq}}\right)^{2}+\Delta^{2}} \hat{\boldsymbol{h}}_{y}^{T}
\end{array}\right] .
$$

The next lemma characterizes the stability properties of the nominal system

$$
\left[\begin{array}{c}
\dot{z}_{1} \\
\dot{z}_{2} \\
\dot{v}_{r}
\end{array}\right]=\boldsymbol{A}\left(z_{2}, u_{r}, u_{c}\right)\left[\begin{array}{c}
z_{1} \\
z_{2} \\
v_{r}
\end{array}\right]
$$

Lemma 1. Under the conditions of Theorem 1, the nominal system (39) is UGAS and ULES.

Proof. To analyze the stability properties of the nominal system (39), we consider the time-derivative of the positive definite and radially unbounded LFC

$$
V \triangleq \frac{1}{2} \sigma^{2}\left|z_{1}\right|^{2}+\frac{1}{2}\left|z_{2}\right|^{2}+\frac{1}{2} \kappa\left|v_{r}\right|^{2}, \quad \kappa>0,
$$

along the solutions of system (39). This gives

$$
\begin{aligned}
\dot{V} \leq & -\sigma^{3} \frac{\Delta\left|z_{1}\right|^{2}}{\left(z_{2}+\sigma y_{\text {int }}^{\text {eq }}\right)^{2}+\Delta^{2}} \\
& -\Delta\left(\frac{\left(U_{d}-V_{\max }\right)-\sigma}{\left(z_{2}+\sigma y_{\text {int }}^{\text {eq }}\right)^{2}+\Delta^{2}}\right)\left|z_{2}\right|^{2} \\
& +\frac{\Delta}{\sqrt{\left(z_{2}+\sigma y_{\text {int }}^{\text {eq }}\right)^{2}+\Delta^{2}}}\left|z_{2}\right|\left|v_{r}\right| \\
& +\kappa \frac{\sigma^{2} \Delta^{2} \bar{X}_{\max }}{\left(\left(z_{2}+\sigma y_{\text {int }}^{\text {eq }}\right)^{2}+\Delta^{2}\right)^{2}}\left|v_{r}\right|\left|z_{1}\right| \\
& +\kappa \frac{\Delta^{2} \bar{X}_{\text {max }}}{\left(z_{2}+\sigma y_{\text {int }}^{\text {eq }}\right)^{2}+\Delta^{2}} \frac{\left(U_{d}+V_{\text {max }}\right)+\sigma}{\left(z_{2}+\sigma y_{\text {int }}^{\text {eq }}\right)^{2}+\Delta^{2}}\left|z_{2}\right|\left|v_{r}\right| \\
& -\kappa\left(Y_{\min }-\frac{\Delta^{2} \bar{X}_{\max }}{\left(\left(z_{2}+\sigma y_{\text {int }}^{\text {eq }}\right)^{2}+\Delta^{2}\right)^{3 / 2}}\right)\left|v_{r}\right|^{2}, \quad(41)
\end{aligned}
$$

after using the notation (19) and Assumption A.4. To simplify the above expressions, we define $\bar{z}_{1} \triangleq\left|z_{1}\right| / \sqrt{\left(z_{2}+\sigma y_{\text {int }}^{\text {eq }}\right)^{2}+\Delta^{2}}$ and $\bar{z}_{2} \triangleq$ $\left|z_{2}\right| / \sqrt{\left(z_{2}+\sigma y_{\text {int }}^{\text {eq }}\right)^{2}+\Delta^{2}}$. With this notation, $\dot{V}$ can be bounded according to

$$
\begin{aligned}
\dot{V} \leq & -\left(\Delta \sigma^{3}-\kappa \frac{\sigma^{2}}{2 \Delta^{2}} \bar{X}_{\max }\right)\left|\bar{z}_{1}\right|^{2} \\
& -\Delta\left(U_{d}-V_{\max }-\sigma\right)\left|\bar{z}_{2}\right|^{2} \\
& +\Delta\left(1+\kappa \frac{\bar{X}_{\max }}{\Delta^{2}}\left(U_{d}+V_{\max }+\sigma\right)\right)\left|\bar{z}_{2}\right|\left|v_{r}\right| \\
& -\kappa\left(Y_{\min }-\frac{1}{\Delta} \bar{X}_{\max }-\frac{\sigma^{2}}{2 \Delta} \bar{X}_{\max }\right)\left|v_{r}\right|^{2} .
\end{aligned}
$$

Furthermore, choosing $\kappa$ equal to (we will show that $\kappa>0$ in the following)

$$
\kappa \triangleq \frac{2 \alpha-1}{\frac{\bar{X}_{\max }}{\Delta^{2}}\left(U_{d}+V_{\max }+\sigma\right)},
$$

where $\alpha$ is given by

$$
\alpha \triangleq\left(U_{d}-V_{\max }-\sigma\right) \frac{\Delta Y_{\min }-\bar{X}_{\max }-\frac{\sigma^{2}}{2} \bar{X}_{\max }}{\bar{X}_{\max }\left(U_{d}+V_{\max }+\sigma\right)},
$$

gives the following bound for $\dot{V}$ :

$$
\begin{gathered}
\dot{V} \leq-\left(\Delta \sigma^{3}-\kappa \frac{\sigma^{2}}{2 \Delta^{2}} \bar{X}_{\max }\right)\left|\bar{z}_{1}\right|^{2} \\
-\Delta\left(U_{d}-V_{\max }-\sigma\right)\left|\bar{z}_{2}\right|^{2}+2 \Delta \alpha\left|\bar{z}_{2}\right|\left|v_{r}\right| \\
-\Delta \frac{\alpha(2 \alpha-1)}{U_{d}-V_{\max }-\sigma}\left|v_{r}\right|^{2}
\end{gathered}
$$

Defining $\beta \triangleq U_{d}-V_{\max }-\sigma$ (we will show that $\beta>0$ in the following), we can finally bound $\dot{V}$ according to

$$
\begin{aligned}
\dot{V} & =-\left(\Delta \sigma^{3}-\kappa \frac{\sigma^{2}}{2 \Delta^{2}} \bar{X}_{\max }\right)\left|\bar{z}_{1}\right|^{2} \\
& -\left[\begin{array}{ll}
\left|\bar{z}_{2}\right| & \left|v_{r}\right|
\end{array}\right]\left[\begin{array}{cc}
\Delta \beta & -\Delta \alpha \\
-\Delta \alpha & \Delta \frac{\alpha(2 \alpha-1)}{\beta}
\end{array}\right]\left[\begin{array}{l}
\left|\bar{z}_{2}\right| \\
\left|v_{r}\right|
\end{array}\right] \\
& \triangleq-W\left(\left|\bar{z}_{1}\right|,\left|\bar{z}_{2}\right|,\left|v_{r}\right|\right) .
\end{aligned}
$$

To guarantee that $W\left(\left|\bar{z}_{1}\right|,\left|\bar{z}_{2}\right|,\left|v_{r}\right|\right)$ is positive definite, or equivalently that $\dot{V}$ is negative definite, we must guarantee that $\alpha>1, \beta>0$ and $\sigma>\kappa /(2 \Delta) \bar{X}_{\max }$. By simple manipulation, one can easily check that condition (21) implies that $\alpha>1$. Note that this also implies that $\kappa>0$, as required for $V$ to be positive definite. Furthermore, $\beta>0$ is guaranteed by Assumption A.3 and the upper bound on $\sigma$ from (22). To check that $\sigma>\kappa /(2 \Delta) \bar{X}_{\max }$, notice that $\kappa$ can be upper bounded according to

$$
\kappa<\frac{2 \alpha}{\frac{\bar{X}_{\max }}{\Delta^{2}}\left(U_{d}+V_{\max }\right)}<2 \Delta^{3} Y_{\min } \frac{U_{d}-V_{\max }-\sigma}{\bar{X}_{\max }^{2}\left(U_{d}+V_{\max }\right)^{2}} .
$$

Using this upper bound on $\kappa$ and the lower bound on $\sigma$ from (22) one can easily check that $\sigma>\kappa /(2 \Delta) \bar{X}_{\max }$. Hence, $\dot{V}$ is negative definite and it follows, from standard Lyapunov arguments, that the nominal system (39) is UGAS. At the same time, note that the function $W$ 


$$
\boldsymbol{A}(z)_{2} \triangleq\left[\begin{array}{ccc}
-\frac{\sigma \Delta}{\left(z_{2}+\sigma y_{\text {int }}^{\mathrm{eq}}\right)^{2}+\Delta^{2}} & \frac{\Delta}{\left(z_{2}+\sigma y_{\text {int }}^{\mathrm{eq}}\right)^{2}+\Delta^{2}} & 0 \\
-\frac{\sigma^{2} \Delta}{\left(z_{2}+\sigma y_{\text {int }}^{\mathrm{eq}}\right)^{2}+\Delta^{2}} & -\Delta \frac{\left(U_{d}+V_{x}\right)-\sigma}{\left(z_{2}+\sigma y_{\mathrm{int}}^{\mathrm{eq}}\right)^{2}+\Delta^{2}} & \frac{\Delta}{\sqrt{\left(z_{2}+\sigma y_{\text {int }}^{\mathrm{eq}}\right)^{2}+\Delta^{2}}} \\
\frac{\sigma^{2} \Delta^{2} \bar{X}\left(u_{r}, u_{c}\right)}{\left(\left(z_{2}+\sigma y_{\text {int }}^{\mathrm{eq}}\right)^{2}+\Delta^{2}\right)^{2}} & \frac{\Delta^{2} \bar{X}\left(u_{r}, u_{c}\right)}{\left(z_{2}+\sigma y_{\text {int }}^{\mathrm{eq}}\right)^{2}+\Delta^{2}} \frac{\left(U_{d}+V_{x}\right)-\sigma}{\left(z_{2}+\sigma y_{\mathrm{int}}^{\mathrm{eq}}\right)^{2}+\Delta^{2}} & \left(Y\left(u_{r}\right)-\frac{\Delta^{2} \bar{X}\left(u_{r}, u_{c}\right)}{\left(\left(z_{2}+\sigma y_{\mathrm{int}}^{\mathrm{eq}}\right)^{2}+\Delta^{2}\right)^{3 / 2}}\right)
\end{array}\right]
$$

satisfies $W\left(\left|\bar{z}_{1}\right|,\left|\bar{z}_{2}\right|,\left|v_{r}\right|\right) \leq \bar{\lambda}_{1}\left|\bar{z}_{1}\right|^{2}+\bar{\lambda}_{2}\left|\bar{z}_{2}\right|^{2}+\lambda_{3}\left|v_{r}\right|^{2}$, for some constants $\bar{\lambda}_{1}, \bar{\lambda}_{2}, \lambda_{3}>0$. Moreover, in any ball $\mathcal{B}_{r}\left\{\left|z_{2}\right| \leq r\right\}, r>0$, the function $W\left(\left|\bar{z}_{1}\right|,\left|\bar{z}_{2}\right|,\left|v_{r}\right|\right)$ can be estimated as $W \leq \lambda_{1}\left|z_{1}\right|^{2}+\lambda_{2}\left|z_{2}\right|^{2}+\lambda_{3}\left|v_{r}\right|^{2}$, where $\lambda_{i}=\bar{\lambda}_{i} /\left(\left(r+\sigma y_{\text {int }}^{\text {eq }}\right)^{2}+\Delta^{2}\right), i=1,2$. This, together with the fact that $V$ is a quadratic function of $z_{1}, z_{2}$ and $v_{r}$, implies that the nominal system (39) is uniformly exponentially stable in any ball $\mathcal{B}_{r}$ (see e.g. [9]). This concludes the proof of Lemma 1.

We have thus proved that the nominal system (39) is UGAS and ULES under the conditions of Theorem 1. It now remains to analyze the interconnected system of (36) and the dynamics of $\boldsymbol{\xi}$, i.e. systems (13a), (17a) and to show that the control goals (7)-(9) are achieved. The next lemma shows that the solutions of system (36) are globally bounded under the perturbation from the $\mathcal{L}_{2}$-signal $\boldsymbol{\xi}(t)$.

Lemma 2. Under the conditions of Theorem 1, the solutions of system (36) are globally bounded.

Proof. The proof of this lemma is given in Appendix B.

According to Lemma 2, the solutions $z_{1}(t), z_{2}(t)$ and $v_{r}(t)$ of system (36) are globally bounded. Then, since $\boldsymbol{\xi}(t) \rightarrow 0$ asymptotically as $t \rightarrow \infty$, it follows that $z_{1}(t) \rightarrow 0, z_{2}(t) \rightarrow$ 0 and $v_{r}(t) \rightarrow 0$ asymptotically as $t \rightarrow \infty$ (see [10]).

Since both $z_{1}$ and $z_{2}$ converges asymptotically to zero, it follows from (33b) that $y$ converges asymptotically to zero. Hence, it follows that control goal (7) is achieved. Moreover, using (31) in the expression

$$
\psi=\psi_{d}+\tilde{\psi}=-\tan ^{-1}\left(\frac{z_{2}+\sigma y_{\mathrm{int}}^{\mathrm{eq}}}{\Delta}\right)+\tilde{\psi}
$$

and using the fact that $z_{2} \rightarrow 0, \tilde{\psi} \rightarrow 0$ asymptotically as $t \rightarrow \infty$, it follows that

$$
\lim _{t \rightarrow \infty} \psi(t)=-\tan ^{-1}\left(\frac{V_{y}}{U_{d}+V_{x}}\right)=\psi_{s s}^{*} .
$$

Hence, control goal (8) is achieved with $\psi_{s s}$ given by $\psi_{s s}^{*}$, cf. Eq. (23).

Next we show that control goal (9) is achieved. Utilizing the expression (29) for $v_{c}$ and using that $\tilde{u} \rightarrow 0, v_{r} \rightarrow 0$ and that $\psi \rightarrow \psi_{s s}^{*}$, we have that

$$
\begin{aligned}
\lim _{t \rightarrow \infty} U(t) & =\lim _{t \rightarrow \infty} \sqrt{\left(u_{d}+\tilde{u}\right)^{2}+\left(v_{r}+v_{c}\right)^{2}} \\
& =\sqrt{\left(U_{d} \cos \psi_{s s}^{*}\right)^{2}+\left(-V_{x} \sin \psi_{s s}^{*}+V_{y} \cos \psi_{s s}^{*}\right)^{2}} .
\end{aligned}
$$

Inserting the expression (23) for $\psi_{s s}^{*}$ in the above equation and rearranging then gives

$$
\lim _{t \rightarrow \infty} U(t)=\sqrt{\frac{U_{d}^{2}\left(V_{y}^{2}+\left(U_{d}+V_{x}\right)^{2}\right)}{V_{y}^{2}+\left(U_{d}+V_{x}\right)^{2}}}=U_{d} .
$$

Hence, control goal (9) is also achieved. This concludes the proof of Theorem 1 .

\section{CONCLUSIONS}

In this paper, we have considered the development of a control strategy for path following of underactuated marine surface vessels in the presence of constant, irrotational ocean currents. In particular, we have proposed a control strategy based on a modified LOS guidance law with integral action and a pair of adaptive tracking controllers. This approach preserved the simplicity and interpretation of traditional LOS guidance, while ensuring sufficient countermeasures against environmental disturbances. The closed-loop dynamics was analyzed in detail and explicit conditions for global asymptotic path following were derived.

\section{APPENDIX A}

$$
\begin{aligned}
& \phi_{u}(\psi, r)=\left[\frac{d_{11}}{m_{11}} \cos \psi-\frac{m_{11}^{A}-m_{22}^{A}}{m_{11}} r \sin \psi,\right. \\
& \left.\frac{d_{11}}{m_{11}} \sin \psi+\frac{m_{11}^{A}-m_{22}^{A}}{m_{11}} r \cos \psi\right]^{T}, \\
& X\left(u_{r}, u_{c}\right) \triangleq \frac{1}{\Gamma}\left[m_{33}\left(-d_{23}-m_{11} u_{r}-m_{11}^{R B} u_{c}\right)\right. \\
& \left.+m_{23} d_{33}+m_{23}\left(m_{23} u_{r}+m_{23}^{R B} u_{c}+m_{22}^{A} u_{c}\right)\right] \\
& Y\left(u_{r}\right) \triangleq \frac{1}{\Gamma}\left[-m_{33} d_{22}+m_{23} d_{32}\right. \\
& \left.+m_{23}\left(m_{22}^{A}-m_{11}^{A}\right) u_{r}\right], \\
& F_{r}(u, v, r) \triangleq \frac{m_{22}}{\Gamma}\left[-\left(m_{22} v-m_{23} r\right) u+m_{11} u v\right. \\
& \left.-d_{32} v-d_{33} r\right]-\frac{m_{23}}{\Gamma}\left[-m_{11} u r-d_{22} v-d_{23} r\right] .
\end{aligned}
$$

Here, $\Gamma \triangleq m_{22} m_{33}-m_{23}^{2}>0$. Furthermore, the function $\phi_{r}(u, v, r, \psi) \triangleq\left[\phi_{r 1}, \ldots, \phi_{r 5}\right]^{T}$ is defined by:

$$
\begin{aligned}
{\left[\begin{array}{c}
\phi_{r 1} \\
\phi_{r 2}
\end{array}\right] } & =\left[\begin{array}{cc}
\cos \psi & -\sin \psi \\
\sin \psi & \cos \psi
\end{array}\right]\left[\begin{array}{l}
a_{1} \\
a_{2}
\end{array}\right] \\
\phi_{r 3} & =-\frac{m_{22}}{\Gamma}\left(m_{11}^{A}-m_{22}^{A}\right) \sin \psi \cos \psi, \\
\phi_{r 4} & =\frac{m_{22}}{\Gamma}\left(m_{11}^{A}-m_{22}^{A}\right) \sin \psi \cos \psi, \\
\phi_{r 5} & =\frac{m_{22}}{\Gamma}\left(m_{11}^{A}-m_{22}^{A}\right)\left(1-2 \sin ^{2} \psi\right),
\end{aligned}
$$


Here,

$a_{1}=-\frac{m_{22}}{\Gamma}\left(\left(m_{11}^{A}-m_{22}^{A}\right) v+\left(m_{23}^{A}-m_{22}^{A}\right) r\right)-\frac{m_{23}}{\Gamma} m_{11}^{A} r$, $a_{2}=\frac{m_{22}}{\Gamma}\left(d_{32}-\left(m_{11}^{A}-m_{22}^{A}\right) u\right)-\frac{m_{23}}{\Gamma} d_{22}$.

Remark: In deriving the expression (52c) we have used that $m_{11}^{R B}-m_{22}^{R B}=0$, which follows from the fact that $m_{11}^{R B}=m_{22}^{R B}=m$, where $m$ is the mass of the vessel (see [6]).

The functions $\boldsymbol{h}_{y} \triangleq\left[h_{y 1}, h_{y 2}, h_{y 3}\right]^{T}$ and $\boldsymbol{h}_{v_{r}} \triangleq$ $\left[h_{v_{r} 1}, h_{v_{r} 2}, h_{v_{r} 3}\right]^{T}$ are given by

$$
\begin{aligned}
h_{y_{1}}= & -\sin \psi_{d}, h_{y_{3}}=0, \\
h_{y_{2}}= & \frac{\cos \tilde{\psi}-1}{\tilde{\psi}}\left(-\left(\tilde{u}+u_{d}\right) \sin \psi_{d}+\left(v_{r}+v_{c}\right) \cos \psi_{d}\right) \\
& -\frac{\sin \tilde{\psi}}{\tilde{\psi}}\left(\left(\tilde{u}+u_{d}\right) \cos \psi_{d}+\left(v_{r}+v_{c}\right) \sin \psi_{d}\right), \\
h_{v r_{1}}= & 0, h_{v_{r} 2}=-\lambda \bar{X}\left(u_{r}, u_{c}\right), h_{v_{r} 3}=\bar{X}\left(u_{r}, u_{c}\right) .
\end{aligned}
$$

The functions $\hat{\boldsymbol{h}}_{y} \triangleq\left[\hat{h}_{y 1}, \hat{h}_{y 2}, \hat{h}_{y 3}\right]^{T}$ is given by

$$
\begin{aligned}
\hat{h}_{y 1}=h_{y 1}, \quad \hat{h}_{y 3}=h_{y 3}, \\
\hat{h}_{y 2}=h_{y 2}+\frac{\sin \tilde{\psi}}{\tilde{\psi}}\left(-V_{x} \cos \psi_{d}-V_{y} \sin \psi_{d}\right) \\
+\frac{\cos \tilde{\psi}-1}{\tilde{\psi}}\left(-V_{x} \cos \sin _{d}+V_{y} \cos \psi_{d}\right) .
\end{aligned}
$$

\section{Appendix B: Proof of LemMa 2}

The perturbation $\boldsymbol{\xi}(t)$ is a vanishing perturbation to the system (36). In particular, $\boldsymbol{\xi}(t) \rightarrow 0$ asymptotically as $t \rightarrow \infty$ and $\boldsymbol{\xi}(t) \in \mathcal{L}_{2}$, cf. Sections IV-C and IV-D. Hence, for any $\epsilon>0$, there exists $t^{*} \geq t_{0}+T$, where $T$ is not necessarily independent of $t_{0}$, such that $\|\boldsymbol{\xi}(t)\| \leq \epsilon, \forall t \geq t^{*}$. Moreover, the time-derivative of the LFC (40) along the solutions of (36) can then be upper bounded by

$$
\dot{V} \leq \frac{c_{2}}{c_{1}} V \cdot\|\boldsymbol{H}\| \cdot\|\boldsymbol{\xi}\| \leq \frac{c_{2}}{c_{1} \epsilon} V \cdot\|\boldsymbol{H}\| \cdot\|\boldsymbol{\xi}\|^{2}, t \in\left[t_{0}, t^{*}\right],
$$

where $c_{1} \leq 1 / 2 \min \left\{\sigma^{2}, 1, \kappa\right\}$ and $c_{2} \geq 1 / 2 \max \left\{\sigma^{2}, 1, \kappa\right\}$. Using Equations (57), (58) and (37), it is straightforward to verify that the interconnection term $\boldsymbol{H}$ is globally bounded, i.e. that $\|\boldsymbol{H}\| \leq b_{H}$, for some $b_{H}>0$. Using this, and integrating both sides of (59) from $t_{0}$ to $t^{*}$ gives

$$
\frac{c_{1} \epsilon}{c_{2} b_{H}} \ln \left(\frac{V\left(t^{*}\right)}{V\left(t_{0}\right)}\right) \leq \int_{t_{0}}^{t^{*}}\|\boldsymbol{\xi}(s)\|^{2} d s \leq \int_{t_{0}}^{\infty}\|\boldsymbol{\xi}(s)\|^{2} d s .
$$

Now, since $\boldsymbol{\xi}(t) \in \mathcal{L}_{2}$, the right hand side of (60) is bounded and it follows that $V(t)$ is bounded on the interval $\left[t_{0}, t^{*}\right]$. In particular, there exits a constant $c \geq 0$ such that $V\left(t^{*}\right)=$ $V\left(\boldsymbol{x}\left(t^{*}\right)\right) \leq c$, where $\boldsymbol{x} \triangleq\left[z_{1}, z_{2}, v_{r}\right]^{T}$. Using Eq. (47), the time-derivative of $V$ can be see to satisfy

$$
\begin{aligned}
\dot{V} & \leq-W\left(\left|\bar{z}_{1}\right|,\left|\bar{z}_{2}\right|,\left|v_{r}\right|\right)+2 c_{2}\|\boldsymbol{x}\| b_{H} \epsilon, \quad t \geq t^{*}, \\
& \leq-\frac{W(\boldsymbol{x})}{\|\boldsymbol{x}\|^{2}+\Delta^{2}}+2 c_{2}\|\boldsymbol{x}\| b_{H} \epsilon, \quad t \geq t^{*} .
\end{aligned}
$$

Note that $W(\boldsymbol{x}) \geq k_{1}\|\boldsymbol{x}\|^{2}$ for some $k_{1}>0$, cf. Eq. (47). Thus,

$$
\begin{aligned}
\dot{V} & \leq-\frac{1}{2} \frac{W(\boldsymbol{x})}{\|\boldsymbol{x}\|^{2}+\Delta^{2}}-\frac{1}{2} \frac{k_{1}\|\boldsymbol{x}\|^{2}}{\|\boldsymbol{x}\|^{2}+\Delta^{2}}+2 c_{2}\|\boldsymbol{x}\| b_{H} \epsilon \\
& \leq-\frac{1}{2} \frac{W(\boldsymbol{x})}{\|\boldsymbol{x}\|^{2}+\Delta^{2}}, \quad \forall \epsilon \leq \frac{k_{1}}{4 c_{2} b_{H}} .
\end{aligned}
$$

Hence, $\dot{V}$ is negative on $V(\boldsymbol{x})=c$, and the set $\{V(\boldsymbol{x})=c\}$ is positively invariant. Thus, for all $\boldsymbol{x}\left(t^{*}\right) \in\left\{c_{2}\|\boldsymbol{x}\|^{2} \leq c\right\}$, $\boldsymbol{x}(t)$ is bounded for all $t \geq t^{*}$. Moreover, since $c>0$ is arbitrary, $\boldsymbol{x}(t)$ is globally bounded. This concludes the proof.

\section{REFERENCES}

[1] A. Healey and D. Lienard, "Multivariable sliding-mode control for autonomous diving and steering of unmanned underwater vehicles," IEEE Journal of Oceanic Engineering, vol. 18, no. 3, pp. 327-339, July 1993.

[2] K. Y. Pettersen and E. Lefeber, "Way-point tracking control of ships," in Proc. of 40th IEEE Conference on Decision and Control, Dec 2001, pp. 940-945.

[3] T. I. Fossen, M. Breivik, and R. Skjetne, "Line-of-sight path following of underactuated marine craft," in Proc. 6th IFAC Manoeuvring and Control of Marine Craft, Girona, Spain, Sep 2003, pp. 244-249.

[4] M. Breivik and T. I. Fossen, "Path following for marine surface vessels," in Proc. MTS/IEEE OCEANS '04, 2004, pp. 2282-2289.

[5] E. Fredriksen and K. Y. Pettersen, "Global $\kappa$ exponential way-point maneuvering of ships: Theory and experiments," Automatica, vol. 42, no. 4, pp. 677687, 2006.

[6] T. I. Fossen, Marine Control Systems. Trondheim, Norway: Marine Cybernetics, 2002.

[7] A. Loria, R. Kelly, and A. R. Teel, "Uniform parametric convergence in the adaptive control of manipulators: a case restudied," in Proc. ICRA '03. IEEE International Conference on Robotics and Automation. IEEE, Sep 2003, pp. 1062-1067.

[8] A. Pavlov, E. Børhaug, E. Panteley, and K. Y. Pettersen, "Straight line path following for formations of underactuated surface vessels," in Proc. IFAC NOLCOS, Pretoria, South Africa, 2007, pp. 654-659.

[9] H. Khalil, Nonlinear Systems, 3rd ed. Upper Saddle River, NJ, USA: Pearson Education International inc., 2000.

[10] E. Sontag, "A remark on the converging-input converging-state property," IEEE Transactions on Automatic Control, vol. 48, no. 2, pp. 313-314, Feb 2003. 